\title{
Short communication: Examination of milk filters by real-time PCR as a herd-level indicator of the presence of Mycobacterium avium subspecies paratuberculosis in dairy herds
}

\author{
I. Slana, ${ }^{1}$ P. Kralik, A. Kralova, V. Babak, and I. Pavlik \\ Veterinary Research Institute, Hudcova 70, 62100 Brno, Czech Republic
}

\begin{abstract}
The aim of this study was to assess the suitability of real-time quantitative PCR (qPCR) for the detection of Mycobacterium avium ssp. paratuberculosis (MAP) in milk filters as a herd level indicator of paratuberculosis infection. Seventy-nine samples from textile or metal milk filters from 15 herds with defined MAP prevalence (infection status $=$ noninfected, $0-5 \%, 5-10 \%$, or $>10 \%$ of animals with clinically confirmed paratuberculosis) were analyzed. The MAP DNA was isolated by a modified commercially available protocol for feces, and detection and quantification of the pathogen was performed by the IS 900 qPCR. Mycobacterium avium ssp. paratuberculosis DNA was detected in $63(79.7 \%)$ samples. Determination of MAP infection established by fecal and tissue culture was correctly confirmed by the analysis of milk filters on 11 of 12 infected farms; MAP was not detected in filters from 3 farms where paratuberculosis was never diagnosed. Statistical analysis of the data supports the evidence that milk filters can be used as a template for the direct detection of MAP on the herd level. The probability of successful MAP detection in milk filters in a herd with MAP-infected cows is at least $94.3 \%$. Absolute numbers of MAP detected on the milk filter can be used for a rough estimation of paratuberculosis prevalence $>10 \%$ in the herd. Analysis of milk filters for the presence of MAP can be a useful tool for the detection of paratuberculosis on the herd level before any individual control strategies.
\end{abstract}

Key words: milk product, food safety, PCR

\section{Short Communication}

Mycobacterium avium ssp. paratuberculosis (MAP) is the causative agent of Johne's disease in ruminants. It can be transmitted by the fecal-oral route to susceptible animals (Ayele et al., 2001). Evidence shows that MAP is detected not only in feces, but also in semen

Received June 27, 2011.

Accepted October 30, 2011.

${ }^{1}$ Corresponding author: slana@vri.cz and tissues of clinically and subclinically infected animals (Khol et al., 2010). Recently, an increasing number of studies have focused on the presence of MAP in milk and milk products (Slana et al., 2008b). The number of MAP cells secreted in milk is $<100 \mathrm{cfu} / \mathrm{mL}$ from clinically infected cows and from 2 to $8 \mathrm{cfu} / 50 \mathrm{~mL}$ in subclinically infected cows (Sweeney et al., 1992; Giese and Ahrens, 2000). Published data suggest that MAP may survive the pasteurization of milk (MAP has been isolated from pasteurized milk) and therefore consumers are exposed to it. This has led to an increase of milk and milk products being tested for the presence of this organism (Grant et al., 2001).

To obtain information about MAP infection status on a herd level, the testing of bulk tank milk (BTM) samples on dairy farms may be a good option to reduce costs compared with the testing of individual animals. Mycobacterium avium ssp. paratuberculosis can be detected by cultivation on Herrold's egg yolk medium or Middlebrook medium supplemented with Mycobactin J or, more recently, by PCR-based techniques targeting IS 900 and F57 or other MAP-specific elements (Gao et al., 2005; Slana et al., 2008a).

The aim of this study was to assess suitability of textile and metal filters for the herd level detection of MAP by quantitative real-time PCR (qPCR) in 15 dairy cattle farms with known MAP infection status.

Textile or metal milk filters are part of milking devices and are used to prevent coarse foreign matter (e.g., hay, feces, hair, cell clumps, and clots present in raw milk) from migrating to the milk tanks. Disposable textile milk filters are made from nonwoven synthetic fibers and are produced as socks. The milk filter (sock) is pulled onto the hollow stainless steel rod and raw milk flows through the filter and the rod to the tank. The textile filter has only limited capacity and it should be changed after 1,500 L of milk or at the end of milking procedure. On the other hand, metal milk filters are based on the filtration of raw milk through a fine sieve (with pores approximately $1 \mathrm{~mm}$ in diameter) made from stainless steel. The sieve is washed and disinfected after each milking cycle and reused. 
The whole textile milk filters and scrapings from the metal sieves were collected irregularly from 3 noninfected and 12 infected dairy farms of different sizes involved in a national paratuberculosis control program in the Czech Republic (Pavlik et al., 2000a). The program was based on fecal culture examination, and most farms had been in the control program for more than $5 \mathrm{yr}$ at the time of sample collection. All animals older than 18 mo were tested. Yearly prevalence of MAP was determined by fecal culture and confirmed by tissue culture examination of ileal mucosa and corresponding lymph nodes (Pavlik et al., 2000b). Textile filters and sieve scrapings were placed in sterile plastic bags and containers, respectively, and chilled at 4 to $6^{\circ} \mathrm{C}$ until further processing.

Total DNA isolation from textile milk filters and metal filter scrapings was based on a protocol of QIAamp DNA Stool Mini Kit (Qiagen, Hilden, Germany) with modifications described for MAP (Kralik et al., 2011). The center of the textile filter was cut to strips of around $5 \mathrm{~mm}$ wide and $1.5 \mathrm{~cm}$ long. Ten such strips (equal to $1 \mathrm{~g}$ ) were added into each of 2 separate 2 -mL screw cap tubes. Similarly, $0.25 \mathrm{~g}$ of metal filter scrapings (removed from the sieve with a sterile spatula) was weighed and added to each tube. Seven hundred milligrams of 1-mm zirconia-silica beads (Biospec, Bartlesville, OK) and $750 \mu \mathrm{L}$ of ASL buffer (provided with the QIAamp kit) were added to each tube, mechanically homogenized in the MagNA Lyser (Roche Molecular Diagnostic, Mannheim, Germany) at $7,000 \mathrm{rpm}$ for $60 \mathrm{~s}$, and then incubated at $99^{\circ} \mathrm{C}$ for 5 min with shaking at 1,400 rpm (Thermomixer Comfort, Eppendorf, Hamburg, Germany).

The entire volume of the liquid phase from each duplicate tube was combined into a single tube containing $350 \mathrm{mg}$ of 0.1-mm zirconia-silica beads (Biospec) and homogenized in the MagNA Lyser (Roche) at $7,000 \mathrm{rpm}$ for $60 \mathrm{~s}$. After removal of PCR inhibitors by the InhibitEX (Qiagen) tablet, protein lysis, and DNA precipitation, the whole volume of suspension was transferred to a spin column. The resulting DNA was eluted using $40 \mu \mathrm{L}$ of Tris-EDTA buffer ( $\mathrm{pH} 8.0$; Amresco, Solon, $\mathrm{OH}$ ) preheated to $70^{\circ} \mathrm{C}$ (Kralik et al., 2011).

The isolated DNA was used as a template for the standardized IS900 TaqMan qPCR assay with a competitive internal amplification control derived from Solanum tuberosum described previously by Slana et al. (2008a). The quantification of MAP in the sample was performed according to the gradient of plasmid standard serially diluted 10 -fold in the range from $5 \times 10^{5}$ to $5 \times 10^{0}$ copies per well. Each sample was analyzed in duplicate by the IS 900 qPCR and was considered positive when one of the replicates gave a positive result.
The quantity of MAP cells present on the textile of metal milk filters was expressed per gram of the input weight of textile or matter scraped from the sieve.

The homogeneity of MAP distribution of the textile filter was checked by analyzing the top, middle, and bottom parts of the milk filter sock.

Statistical analysis of data obtained from analysis of 79 milk filter samples from 15 farms was performed using statistical software Statistica 9 (StatSoft Inc., Tulsa, OK) and GraphPad Prism 5 (GraphPad Software Inc., San Diego, CA). The chi-squared test for independence and Kruskal-Wallis with Dunn's multiple comparison post-hoc test were used analyze the data. The farms were divided into 4 groups according to the prevalence of paratuberculosis (number of animals with clinically confirmed paratuberculosis) in the herd per year: noninfected (0\%), low (0-5\%), moderate (5-10\%), and high $(>10 \%$; Table 1$)$.

Because of their good accessibility without further manipulation of the animals, milk filters could be a suitable tool for quick assessment of the MAP infection status of the dairy herd. The use of milk filters to evaluate the presence of MAP as an indicator of MAP herd prevalence was first noted by McKee et al. (2002). In that study, milk filters were analyzed for the presence of MAP by immunomagnetic separation followed by IS 900 PCR, and MAP DNA was found in $15(8.1 \%)$ of 186 milk filters tested (McKee et al., 2002). In our study, 79 filters from 15 herds were investigated and 63 $(79.7 \%)$ were found to be positive (Table 1$)$. Although the number of samples in our study was smaller, we obtained more complex information about applicability of milk filter analysis in the detection of MAP on the herd level because milk filter samples originated from herds with defined infection status.

A statistically significant association between infectious status of the herd and frequency of MAP-positive filters were found ( $P$-value of chi-squared test for independence $<0.01$ ). No significant difference was revealed among frequencies of MAP-positive filters in herds with low, moderate, and high prevalence of MAP ( $P$-value of chi-squared test for independence $>0.05$ ).

When the tested farms were divided into only noninfected and infected (culture examination of feces and tissues was considered as the gold standard), parameters of the screening method were determined. Diagnostic sensitivity of qPCR examination of milk filters was estimated to be $85.1 \%$ (95\% CI: 75.0 to $92.4 \%$ ) and diagnostic specificity $100 \%$ (95\% CI: 47.9 to $100 \%$ ). Positive predictive value was determined to be $100 \%$ (95\% CI: 94.3 to 100\%). The confidence interval of positive predictive value is interpreted to mean that with a risk of $5 \%$, the probability of successful MAP detection from a milk filter in the herd with MAP- 
Table 1. Detection of Mycobacterium avium subspecies paratuberculosis DNA by the IS900 real-time quantitative PCR (qPCR) on textile and metal milk filters originating from farms differing in paratuberculosis infectious status

\begin{tabular}{|c|c|c|c|c|c|c|c|}
\hline \multirow[b]{2}{*}{ Farm } & \multirow[b]{2}{*}{$\begin{array}{l}\text { Infection } \\
\text { status }\end{array}$} & \multirow[b]{2}{*}{$\begin{array}{l}\text { Filter } \\
\text { type }\end{array}$} & \multirow[b]{2}{*}{$\begin{array}{l}\text { Herd size } \\
\quad \text { (no.) }\end{array}$} & \multicolumn{2}{|c|}{$\begin{array}{c}\text { Animals with } \\
\text { clinically confirmed } \\
\text { paratuberculosis } \\
\text { per year }\end{array}$} & \multicolumn{2}{|c|}{ IS 900 qPCR } \\
\hline & & & & No. & $\%$ & $\begin{array}{c}\text { No. of } \\
\text { samples }\end{array}$ & $\begin{array}{l}\text { No. } \\
\text { positive }\end{array}$ \\
\hline 1 & Noninfected & Textile & 300 & 0 & 0 & 1 & 0 \\
\hline 2 & & Metal & 360 & 0 & 0 & 2 & 0 \\
\hline 3 & & Textile & 620 & 0 & 0 & 2 & 0 \\
\hline 4 & Low & Metal & 350 & 4 & 1.1 & 2 & 1 \\
\hline 5 & & Textile & 140 & 2 & 1.4 & 30 & 28 \\
\hline 6 & & Textile & 73 & 2 & 2.7 & 10 & 9 \\
\hline 7 & & Textile & 505 & 21 & 4.2 & 2 & 1 \\
\hline 8 & & Metal & 250 & 11 & 4.4 & 1 & 0 \\
\hline 9 & Moderate & Textile & 350 & 25 & 7.1 & 2 & 2 \\
\hline 10 & & Textile & 250 & 21 & 8.4 & 8 & 4 \\
\hline 11 & & Textile & 380 & 34 & 8.9 & 1 & 1 \\
\hline 12 & & Textile & 800 & 75 & 9.4 & 2 & 2 \\
\hline 13 & High & Textile & 180 & 20 & 11.1 & 3 & 3 \\
\hline 14 & & Metal & 240 & 45 & 18.8 & 12 & 11 \\
\hline 15 & & Textile & 400 & 84 & 21.0 & 1 & 1 \\
\hline
\end{tabular}

infected cows will be at least $94.3 \%$. The presented method for the determination of MAP in milk filters by qPCR confirmed the presence of paratuberculosis in 11 of 12 infected herds; MAP was not detected in milk filters from noninfected herds.

Distribution of MAP absolute values within farms with positive infection status (low, moderate, or high) did not show a normal distribution even after logarithmic transformation (Figure 1). Kruskal-Wallis test showed that infection status is a statistically significant source of variability of IS 900 values $(P<0.01)$. The subsequently performed Dunn's multiple comparison test (post-hoc test) displayed statistically significant differences between categories low and high and between moderate and high $(P<0.01)$, whereas a statistically significant difference was not demonstrated $(P>0.05)$ between the low and moderate categories. We conclude that the absolute number of MAP on the filter reflects the prevalence of clinically infected animals in the herd; however, this applies only to herds with more than $10 \%$ of animals having clinically confirmed paratuberculosis. Prevalence $<10 \%$ cannot be reliably distinguished using this method.

The MAP distribution on the textile milk filter was homogeneous without any part preference. Sampling of the top, middle, and bottom parts of the textile milk filter did not show any significant deviations. Even in filters from farms with low and moderate numbers of MAP-infected animals, the probability of successful detection from a single sample was $>75 \%$ (data not shown). If duplicate sampling and isolation were per- formed, the probability of detection increased to $95 \%$. Thus, we recommend analyzing more filters, especially in herds with a low number of infected cows, because it could increase the probability of successful detection (Table 1). The frequency of MAP detection in milk filters was not influenced by the size of the herd.

Analysis of textile or metal milk filters is a suitable and easily accessible tool of choice for the direct detection and screening of MAP infection status on the herd level. Collection and analysis of such samples is easy and inexpensive. Compared with samples of BTM or pooled feces, analysis of milk filters is less laborious (no mixing of feces or time-consuming centrifugation of milk) and it uses material that is always discarded. Long-term storage of filters for the analysis is also possible. Problems with reliability of MAP detection can arise when using BTM samples because of the dilution effect of milk. However, examination of milk filters does not provide any more detailed information than pooled fecal samples regarding individual infection status. This method could be used for the basic rapid determination of herd infection status before application of any direct MAP intervention strategies.

\section{ACKNOWLEDGMENTS}

This work was supported by the EC (PathogenCombat No. FOOD-CT-2005-007081), AdmireVet (No. CZ.1.05/2.1.00/01.0006 - ED0006/01/01) of the Ministry of Education, Youth and Sports of the Czech Republic and the Ministry of Agriculture of the Czech 


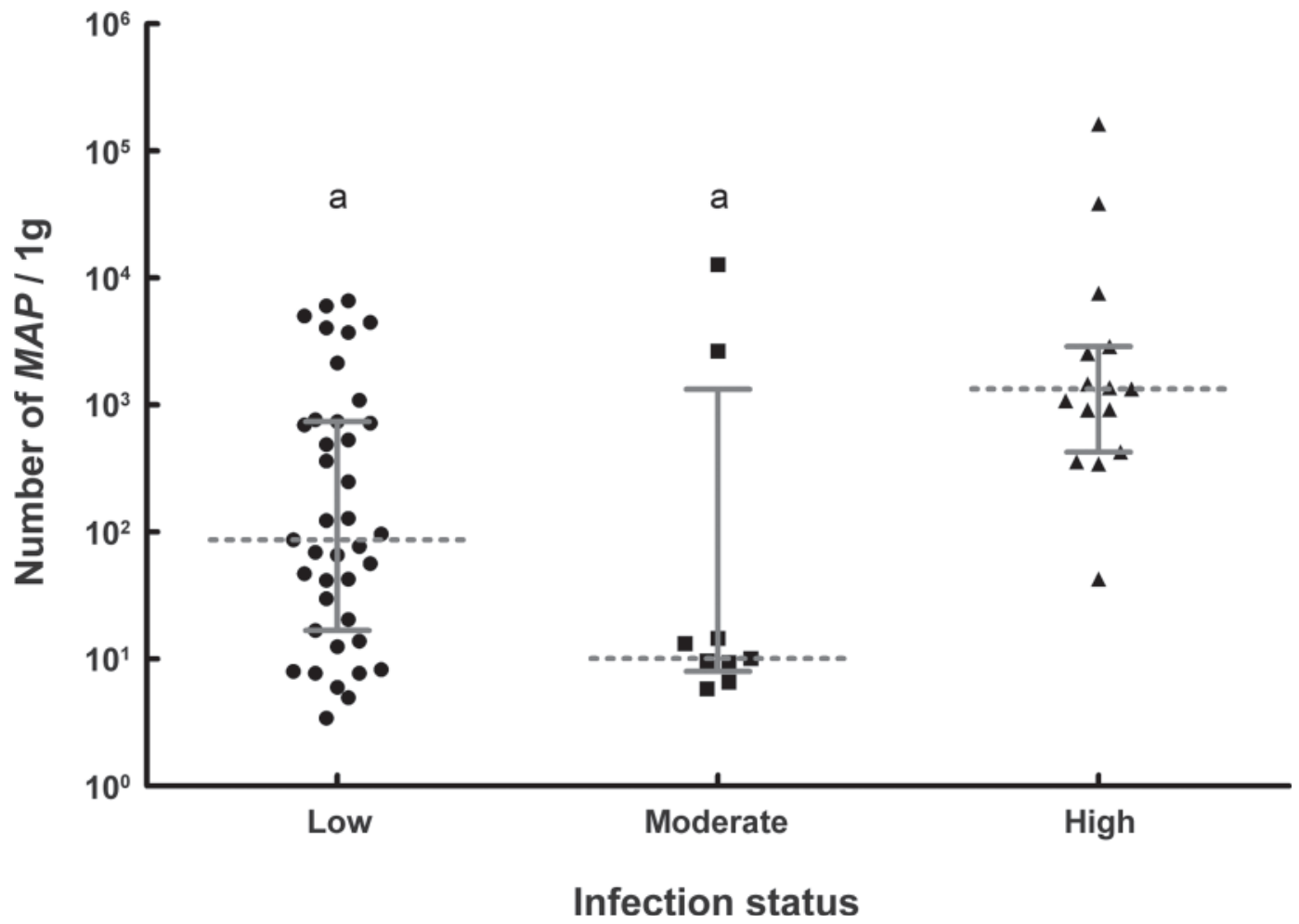

Figure 1. Distribution of absolute numbers of Mycobacterium avium ssp. paratuberculosis (MAP) per gram of milk filter as determined by IS 900 quantitative real-time PCR in farms with different infection status (low $=0-5 \%$, moderate $=5-10 \%$, and high $=>10 \%$ of animals with clinically confirmed paratuberculosis). Dashed lines represent medians of each group, and error bars indicate the lower and upper quartile (25th and 75th percentile). Letters mark selective groups where no statistically significant difference was observed $(P>0.05 ;$ Dunn's multiple comparison test).

Republic (Grants Nos. MZe0002716202 and QH81065). We acknowledge Neysan Donnelly (National University of Ireland, Galway) for the critical English correction of the article.

\section{REFERENCES}

Ayele, W. Y., M. Machackova, and I. Pavlik. 2001. The transmission and impact of paratuberculosis infection in domestic and wild ruminants. Vet. Med. (Czech) 46:205-224.

Gao, A., J. Odumeru, M. Raymond, and L. Mutharia. 2005. Development of improved method for isolation of Mycobacterium avium ssp. paratuberculosis from bulk tank milk: Effect of age of milk, centrifugation, and decontamination. Can. J. Vet. Res. 69:81-87.

Giese, S. B., and P. Ahrens. 2000. Detection of Mycobacterium avium ssp. paratuberculosis in milk from clinically affected cows by PCR and culture. Vet. Microbiol. 77:291-297.

Grant, I. R., M. T. Rowe, L. Dundee, and E. Hitchings. 2001. Mycobacterium avium ssp. paratuberculosis: Its incidence, heat resistance and detection in milk and dairy products. Int. J. Dairy Technol. 54:2-13.

Khol, J. L., P. Kralik, I. Slana, V. Beran, C. Aurich, W. Baumgartner, and I. Pavlik. 2010. Consecutive excretion of Mycobacterium avium subspecies paratuberculosis in semen of a breeding bull compared to the distribution in feces, tissue and blood by IS900 and F57 quantitative real-time PCR and culture examinations. J. Vet. Med. Sci. 72:1283-1288.

Kralik, P., I. Slana, A. Kralova, V. Babak, R. H. Whitlock, and I. Pavlik. 2011. Development of a predictive model for detection of
Mycobacterium avium subsp paratuberculosis in faeces by quantitative real time PCR. Vet. Microbiol. 149:133-138.

McKee, R., I. R. Grant, M. T. Rowe, H. G. Buckley, J. F. Buckley, and S. Fanning. 2002. Examination of in-line filters to detect Mycobacterium avium ssp. paratuberculosis infection at farm level. Page 258-260 in Proc. 7th Int. Colloq. Paratuberculosis. R. A. Juste, M. V. Geijo, and J. M. Garrido, ed. International Association for Paratuberculosis Inc., Madison, WI.

Pavlik, I., L. Matlova, J. Bartl, P. Svastova, L. Dvorska, and R. Whitlock. 2000b. Parallel faecal and organ Mycobacterium avium ssp. paratuberculosis culture of different productivity types of cattle. Vet. Microbiol. 77:309-324.

Pavlik, I., Z. Rozsypalova, T. Vesely, J. Bartl, L. Matlova, V. Vrbas, L. Valent, D. Rajsky, I. Mracko, M. Hirko, and P. Miskovic. 2000a. Control of paratuberculosis in five cattle farms by serological tests and faecal culture during the period 1990-1999. Vet. Med. (Czech) 45:61-70.

Slana, I., P. Kralik, A. Kralova, and I. Pavlik. 2008a. On-farm spread of Mycobacterium avium ssp. paratuberculosis in raw milk studied by IS 900 and $F 57$ competitive real time quantitative PCR and culture examination. Int. J. Food Microbiol. 128:250-257.

Slana, I., F. Paolicchi, B. Janstova, P. Navratilova, and I. Pavlik 2008b. Detection methods for Mycobacterium avium ssp. paratuberculosis in milk and milk products: A review. Vet. Med. (Czech) $53: 283-306$.

Sweeney, R. W., R. H. Whitlock, and A. E. Rosenberger. 1992. Mycobacterium paratuberculosis cultured from milk and supramammary lymph nodes of infected asymptomatic cows. J. Clin. Microbiol. 30:166-171. 